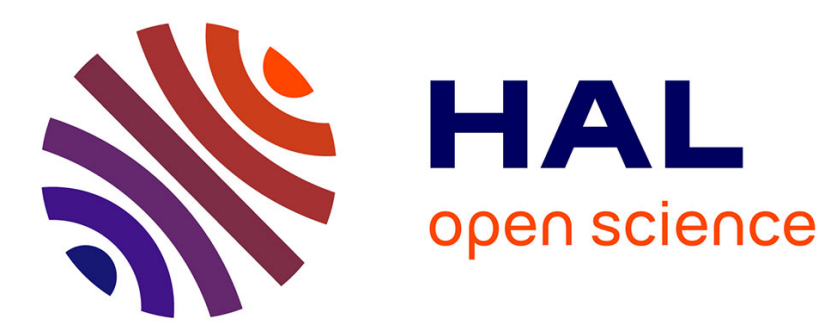

\title{
Observateur non linéaire étendu
}

Ramdane Tami, Driss Boutat, Gang Zheng

\section{To cite this version:}

Ramdane Tami, Driss Boutat, Gang Zheng. Observateur non linéaire étendu. Journal Européen des Systèmes Automatisés (JESA), 2013, 47 (4-8), pp.503-518. 10.3166/HSP.x.1-16 . hal-01094932

\section{HAL Id: hal-01094932 \\ https://hal.inria.fr/hal-01094932}

Submitted on 18 Dec 2014

HAL is a multi-disciplinary open access archive for the deposit and dissemination of scientific research documents, whether they are published or not. The documents may come from teaching and research institutions in France or abroad, or from public or private research centers.
L'archive ouverte pluridisciplinaire HAL, est destinée au dépôt et à la diffusion de documents scientifiques de niveau recherche, publiés ou non, émanant des établissements d'enseignement et de recherche français ou étrangers, des laboratoires publics ou privés. 
2013xy

\title{
Observateur non linéaire étendu
}

\author{
Ramdane Tami $^{1}$, Driss Boutat ${ }^{2}$, Gang Zheng $^{3}$ \\ 1. INSA Centre Val de Loire, univ. d'Orléans, Laboratoire PRISME UPRES 4229, 88 \\ BLD Lahitolle, CS 60013, 18020 Bourges Cedex, France \\ ramdane.tami@insa-cvl.fr \\ 2. INSA Centre Val de Loire, univ. d'Orléans, Laboratoire PRISME UPRES 4229, 88 \\ BLD Lahitolle, CS 60013, 18020 Bourges Cedex, France \\ driss.boutat@insa-cvl.fr \\ 3. INRIA Lille-Nord Europe, 40 Avenue Halley 59650, Villeneuve d'Ascq, France \\ gang.zheng@inria.fr
}

RÉSUMÉ. Cet article étudie le problème de la recherche d'un changement de coordonnées permettant de transformer un système non-linéaire en forme normale d'observabilité, qui est une forme idéale pour l'estimation d'états. Des conditions géométriques suffisantes pour l'existence d'un changement de coordonnées d'un un système non linéaire mono sortie sont données.

ABSTRACT. This paper deals with the observer design for nonlinear dynamical system after transformation through change of coordinates into an observer normal form which supports a high gain observer. Moreover, the sufficient geometrical conditions that guarantee the existante of change of coordinates are given. The proposed approach is applied to estimate the states of SEIR model.

MOTS-CLÉS : estimation d'états, forme normale d'obsrvabilité, dynamique étendue, système nonlinéaire.

KEYWORDS: states estimation, observer normal form, extended dynamics, nonlinear system. 


\section{Introduction}

Le caractère imprévisible des maladies et la mutation des virus nécessite la mise en place doutils permettant lanalyse, la prédiction et le suivi du phénomène de propagation épidémique. Le système dynamique SEIR (Susceptible, Exposed, In- fected, Removed) modélise la propagation dune maladie contagieuse à travers une population. Plusieurs travaux ont été menés pour améliorer ce modèle, étudier sa stabilité et développer des stratégies de vaccination. Cependant, peu de travaux se sont intéressés à la conception dobservateurs pour ce modèle (Iggidr et al., 2006). Par conséquent, se basant sur le modelé SRIR, ce travail vise à construire un observateur pour estimer l'évolution d'une épidémie. Dans cette optique, nous proposons une approche basée sur le concept des formes normales dobservabilité et de la dynamique étendue.

Le concept de forme normale d'observabilité a été introduit par (Krener, Isidori, 1983), (Bestle, Zeitz, 1983) pour une certaine classe de systèmes dynamiques non linéaires mono-sortie, ensuite il a été étendu par (Krener, Respondek, 1985), (Xia, Gao, 1989) aux systèmes dynamiques multi-sorties. A partir de là, plusieurs algorithmes ont été proposés donnant lieu à divers approches: algébrique (Keller, 1987), (Phelps, 1991), (Rudolph, Zeitz, 1994), géométrique (Hou, Pugh, 1999), (Marino, Tomei, 1996), (Boutat et al., 2006), (Lynch, Bortoff, 2001) et les transformations directes (Lopez et al., 1999).

Afin d'élargir la classe des systèmes non-linéaires transformables par diffeomorphisme sous forme normale d'observabilité, (Respondek et al., 2004) proposa la forme normale dépendante de la sortie, consolidée par la suite dans (Zheng et al., 2007), (Wang, Lynch, 2009). Toujours, dans l'optique de pouvoir transformer la plus large classe possible de systèmes non-linéaires sous forme appropriée à l'estimation d'états, (Jouan, 2003) développa la forme normale étendue dont le principe consiste à ajouter une dynamique auxiliaire au système non-linéaire de sorte que le système augmenté resultant satisfasse les conditions de la mise sous forme normale d'observabilité étendue. Cette méthode a été affinée progressivement dans (Noh et al., 2004), (Back et al., 2006), (Yang et al., 2010), (Boutat, 2007), (Boutat, Busawon, 2011).

Malgré la multitudes d'algorithmes proposées permettant la construction des formes normales, il est nécessaire de les améliorer et de les renforcer pour plus d'efficacité face aux non-linéarités d'ordre supérieur. Dans cette optique, la forme normale étendue dépendante de la sortie est mise en oeuvre

Dans ce papier nous proposons une nouvelle forme normale d'observabilité. L'idée consiste à coupler les formes normales étendues avec les formes normales dépendantes de la sortie. L'efficacité de cette approche est démontrée sur un modèle non-linéaire de propagation épidémique SEIR (Susceptible, Exposed, Infected and Removed).

Le caractère imprévisible des maladies et la mutation des virus nécessitent la mise en place d'outils permettant l'analyse, la prédiction et le suivi du phénomène de propagation épidémique. Par conséquent, dans ce papier, un observateur est synthétisé afin d'estimer les différentes populations concernées par l'épidémie et de prédire son 
évolution. L'approche utilisée est basée sur le concept des formes normales d'observabilité et de la dynamique étendue (immersion).

Cet article est organisé comme suit : la section $(I I)$ présente l'exemple de motivation. Un rappel sur la mise sous forme normale d'observabilité est donné dans la section $(I I I)$. La section $(I V)$ est dédiée à la forme normale étendue dépendante de la sortie et les conditions suffisantes pour son obtention. La mise en application de la méthode étudiée et les résultats de simulation sont présentés dans la section $(V)$.

\section{Modèle épidémiologique et forme normale étendue}

Le modèle épidémique SEIR décrit la propagation d'une maladie contagieuse à travers une population, il met en jeu 4 variables: $S(t)$ la population susceptible à la maladie, $E(t)$ la population exposée mais qui ne présente pas encore de symptômes, $I(t)$ la population infectée et $R(t)$ la population traitée. Plus précisément, ce modèle est décrit par le système non linéaire:

$$
\begin{aligned}
\frac{d S}{d t} & =b N-\mu S-\beta \frac{S I}{N}-p b E-q b I \\
\frac{d E}{d t} & =\beta \frac{S I}{N}+p b E+q b I-(\mu+\varepsilon) E \\
\frac{d I}{d t} & =\varepsilon E-(r+\delta+\mu) I \\
\frac{d R}{d t} & =r I-\mu R \\
\frac{d N}{d t} & =(b-\mu) N-\delta I
\end{aligned}
$$

où $N=S+E+I+R$ représente la population totale, $b$ le taux de naissance naturelle, $\mu$ le taux de fécondité, $\beta$ le taux de transmission de l'épidémie, $\delta$ le taux de mortalité épidémique, $\varepsilon$ le taux passage des individus exposés au cas infectés, $p$ le taux de descendance chez la population exposée, $q$ le taux de descendance chez la population infectée et $r$ le taux de guérison.

Afin de faciliter l'écriture des equations, dans la suite de l'article, des variables normalisées sont utilisés, telles que $x_{1}=\frac{S}{N}, x_{2}=\frac{E}{N}, x_{3}=\frac{I}{N}, x_{4}=\frac{R}{N}$ et $x_{1}+$ $x_{2}+x_{3}+x_{4}=1$. Après dérivation de ces nouvelles variables, le modèle SEIR (1)-(4) devient:

$$
\begin{aligned}
\dot{x}_{1} & =b-b x_{1}+\gamma_{1} x_{1} x_{3}-p b x_{2}-q b x_{3} \\
\dot{x}_{2} & =\beta x_{1} x_{3}+\gamma_{2} x_{2}+\delta x_{2} x_{3}+q b x_{3} \\
\dot{x}_{3} & =\varepsilon x_{2}+\gamma_{3} x_{3}+\delta x_{3}^{2} \\
\dot{x}_{4} & =r x_{3}+b x_{4}+\delta x_{3} x_{4} \\
y & =x_{3}
\end{aligned}
$$


øù $\gamma_{1}=-(\beta-\delta), \gamma_{2}=-(b+\varepsilon-p b), \gamma_{3}=-(r+\delta+b)$. Les états $\hat{x}_{1}$ et $\hat{x}_{2}$ seront estimés par un observateur, tandis que l'état $\hat{x}_{4}$ sera déduit à partir de l'equation algébrique $\hat{x}_{4}=1-\hat{x}_{1}-\hat{x}_{2}-x_{3}$.

Dans la section $(V)$, il est montré que le système dynamique (6)-(8) ne satisfait pas les conditions géométriques existantes dans la littérature (Krener, Isidori, 1983), (Krener, Respondek, 1985), (Kazantzis, Kravaris, 1998), (Boutat et al., 2009). Pour remédier à ce problème, une dynamique étendue est ajoutée, qui sera déterminée par la suite, de telle sorte que le nouveau système augmenté satisfasse les conditions suffisantes grandissant l'existence du diffeomorphisme. En effet, si la dynamique auxiliaire

$$
\dot{w}=-b+\gamma_{1} x_{3}
$$

est ajoutée au système (6)-(8), alors le changement de coordonnées $z=\phi(x, w)$ définit par :

$$
\begin{aligned}
z_{1} & =\beta \varepsilon x_{1} e^{-w}+b p \beta x_{3} e^{-w} \\
z_{2} & =e^{-w} x_{2}-\frac{b+\gamma_{2}}{\varepsilon} x_{3} e^{-w}-\frac{\delta-\gamma_{1}}{2 \varepsilon} x_{3}^{2} e^{-w} \\
z_{3} & =\frac{1}{\varepsilon} x_{3} e^{-w} \\
\xi & =w
\end{aligned}
$$

transforme le système (6)-(8) augmenté de la dynamique auxiliaire (11) sous la forme normale étendue dépendante de la sortie donnée par :

$$
\begin{aligned}
\dot{z}_{1} & =B_{1}(w, y) \\
\dot{z}_{2} & =y z_{1}+B_{2}(w, y) \\
\dot{z}_{3} & =z_{2}+B_{3}(w, y) \\
\dot{w} & =B_{4}(w, y) \\
\bar{y} & =z_{3}
\end{aligned}
$$

où la variable $w$ est considérée comme une sortie auxiliaire et les expressions des $B_{i}$ seront données dans la section (5).

Ce papier établit des conditions suffisantes pour l'existence du changement de coordonnées transformant un système non linéaire sous forme normale étendue dépendante de la sortie:

$$
\begin{aligned}
\dot{z} & =A(y) z+B(w, y) \\
\dot{w} & =B_{n+1}(w, y) \\
\bar{y} & =C z
\end{aligned}
$$


avec $C=[0, \ldots, 0,1]$ et

$$
A=\left(\begin{array}{cccc}
0 & \cdots & 0 & 0 \\
\alpha_{2}(y) & \cdots & 0 & 0 \\
\vdots & \ddots & \vdots & \vdots \\
0 & \cdots & \alpha_{n}(y) & 0
\end{array}\right)
$$

Cette forme a une configuration adaptée pour l'utilisation de l'observateur à grand gain (Busawon et al., 1998):

$$
\dot{\widehat{z}}=A(y) \widehat{z}+B(w, y)-\Gamma^{-1}(y) R_{\rho}^{-1} C^{T}(C \widehat{z}-\bar{y})
$$

avec $G, \Gamma$ et $R_{\rho}$ des paramètres donnés par :

$$
\begin{gathered}
G_{n \times n}=\left(\begin{array}{cccc}
0 & \cdots & 0 & 0 \\
1 & \cdots & 0 & 0 \\
\vdots & \ddots & \vdots & \vdots \\
0 & \cdots & 1 & 0
\end{array}\right) \\
\Gamma(y)=\operatorname{diag}\left[\prod_{i=2}^{n} \alpha_{i}(y), \prod_{i=3}^{n} \alpha_{i}(y), \cdots, \alpha_{n}(y), 1\right] \\
R_{\rho}(n+1-i, n+1-j)=\frac{(-1)^{i+j} C_{i+j-2}^{j-1}}{\rho^{i+j-1}}
\end{gathered}
$$

On montre que la dynamique de l'erreur d'observation est donnée par:

$$
\dot{e}=\left(A(y)-\Gamma^{-1}(y) R_{\rho}^{-1} C^{T} C\right) e
$$

Si $y$ et $w$ sont bornées, la dynamique de l'erreur d'observation est exponentiellement stable avec un $\rho$ bien choisi.

Remarque 1. - La dynamique ajoutée (11) ne depend que de la sortie et d'elle même.

\section{Préliminaires}

Considérons un système non linéaire mono sortie:

$$
\begin{aligned}
& \dot{x}=f(x) \\
& y=h(x)
\end{aligned}
$$

où $x \in U \subseteq \mathbb{R}^{n}$ sont les l'état du système et $y \in \mathbb{R}$ est la sortie du système. On suppose que le champ de vecteurs $f$ régissant la dynamique et la fonction de sortie $h$ 
\$ont suffisamment rigoureux (derivables) et que la paire $(h, f)$ est observable, c'est à dire, les 1-formes différentielles

$$
\begin{aligned}
& \theta_{1}=d h \\
& \theta_{i}=d L_{f}^{i-1} h \text { pour } i=2: n
\end{aligned}
$$

sont indépendantes, avec $L_{f}^{k}$ est la $k^{\text {eme }}$ dérivée de Lie de $h$ dans la direction du champ de vecteurs $f$. Cette hypothèse permet de construire le repère de Krener \& Isidori (Krener, Isidori, 1983) $\tau=\left[\tau_{1}, \ldots, \tau_{n}\right]$ où le premier champ de vecteurs $\tau_{1}$ est donné par l'équation algébrique:

$$
\left\{\begin{array}{c}
\theta_{i}\left(\tau_{1}\right)=0 \text { pour } i=1: n-1 \\
\theta_{n}\left(\tau_{1}\right)=1
\end{array}\right.
$$

et les autres champs de vecteurs sont obtenus par induction:

$$
\tau_{i}=-a d_{f} \tau_{i-1}=\left[\tau_{i-1}, f\right] \text { pour } 2 \leq i \leq n,
$$

où le symbole [,] désigne le crochet de Lie. A présent, nous allons énumérer quelques formes normales les plus connues et les conditions garantissant leur exitance. D'après (Krener, Isidori, 1983) si la condition de commutativité est satisfaite:

$$
\left[\tau_{i}, \tau_{j}\right]=0
$$

alors, il existe un changement de coordonnées $z=\phi(x)$ permettant de transformer le système (18)-(19) sous la forme normale d'observabilité

$$
\left\{\begin{array}{c}
\dot{z}=A z+B(y) \\
y=C z
\end{array}\right.
$$

avec $C=[0, \ldots, 0,1]$ et

$$
A=\left(\begin{array}{cccc}
0 & \cdots & 0 & 0 \\
1 & \cdots & 0 & 0 \\
\vdots & \ddots & \vdots & \vdots \\
0 & \cdots & 1 & 0
\end{array}\right)
$$

La condition (22) assurant l'existence d'un changement de coordonnées est relativement difficile à satisfaire.

Dans (Zheng et al., 2007) des conditions moins restrictives ont été proposées pour l'existence du changement de cordonnées. En effet, supposons que le repère $\tau$ vérifie les conditions suivantes:

$$
\left\{\begin{array}{c}
{\left[\tau_{1}, \tau_{n}\right]=\lambda_{1}(y) \tau_{1}} \\
{\left[\tau_{k}, \tau_{n}\right]=\lambda_{k}(y) \tau_{k} \text { modulo Eng }\left\{\tau_{1}, \cdots, \tau_{k-1}\right\}} \\
\text { pour } 2 \leq k \leq n-2
\end{array}\right.
$$


Dès lors, on considère une famille de fonctions non nulles dépendantes de la sortie $\alpha_{2}(y), \ldots, \alpha_{n}(y)$ et soit $\pi=\prod_{i=2}^{n} \alpha_{i}(y)=\alpha_{2}(y) \times \ldots \times \alpha_{n}(y)$. Puis, on construit un nouveau repère comme suit:

$$
\left\{\begin{array}{c}
\bar{\tau}_{1}=\pi \tau_{1} \\
\bar{\tau}_{i}=\frac{1}{\alpha_{i}}\left[\bar{\tau}_{i-1} f\right]
\end{array}\right.
$$

Si le repère $\bar{\tau}$ est commutatif, c'est-à-dire :

$$
\left[\bar{\tau}_{i}, \bar{\tau}_{j}\right]=0
$$

alors il est prouvé dans (Respondek et al., 2004) pour $\alpha_{2}=\ldots=\alpha_{n}$ et pour des $\alpha_{i}$ différents dans (Zheng et al., 2007) que le système dynamique (18)-(19) peut être transformé grâce à un changement de coordonnées $z=\phi(x)$ sous la forme d'observabilité dépendante de la sortie:

$$
\begin{aligned}
\dot{z} & =A(y) z+B(y) \\
y & =C z
\end{aligned}
$$

où $A(y)$ est définie dans (17).

Dans le cas où ni la condition (22), ni la condition (27) ne sont satisfaites, alors on passe à l'algorithme utilisant la dynamique étendue.

\section{Forme normale non linéaire étendue dépendante de la sortie}

Cette section met en évidence des conditions géométriques suffisantes qui garantissent l'existence d'une dynamique auxiliaire $\dot{w}=\eta(y, w)$ telle que le système dynamique augmenté:

$$
\begin{aligned}
\dot{x} & =f(x) \\
\dot{w} & =\eta(y, w) \\
y & =h(x)
\end{aligned}
$$

peut être transformé par un changement de coordonnées $(z, \xi)=\phi(x, w)$ sous la forme normale étendue dépendante de la sortie:

$$
\begin{aligned}
\dot{z} & =A(y) z+B(y, w) \\
\dot{\xi} & =B_{n+1}(y, w) \\
y & =C z
\end{aligned}
$$

où $A(y)$ est défini dans (17). Le cas où la matrice $A(y)$ est constante (24) est largement étudié dans (Back et al., 2006; Jouan, 2003 ; Boutat, 2007 ; Boutat, Busawon, 2011). 
/ Pour ce qui suit, la condition (25) est supposé satisfaite, par conséquent on peut determiner les fonctions $\alpha_{2}(y), \ldots, \alpha_{n}(y)$ ainsi que le repère $\bar{\tau}$ défini dans (26).

Maintenant, définissons un nouveau repère comme suit:

$$
\begin{aligned}
\sigma_{1} & =l(w) \bar{\tau}_{1} \text { où la fonction } l(w) \neq 0 \text { est à déterminer } \\
\sigma_{k} & =\frac{1}{\alpha_{k}}\left[\sigma_{k-1}, F\right]
\end{aligned}
$$

où $F=f+\eta(y, w) \frac{\partial}{\partial w}$ est le champ de vecteurs régissant le système étendue.

Théorème 2. - S'il existe une fonction $l(w) \neq 0$ de la variable auxiliaire $w$ telle que $\left[\sigma_{i}, \sigma_{j}\right]=0$ pour $1 \leq i, j \leq n$, alors il existe un changement de coordonnées $(z, \xi)=\phi(x, w)$ qui permet de transformer le système dynamique étendu (28)-(30) sous la forme normale étendue dépendante de la sortie (31)-(33).

Preuve 3. - Supposons qu'il existe $l(w) \neq 0$ tel que $\left[\sigma_{i}, \sigma_{j}\right]=0$ pour $1 \leq i, j \leq n$. Soit $\sigma_{n+1}$ un champ de vecteurs indépendants de $\sigma_{i}$ pour tout $i=1: n$, tel que $\left[\sigma_{i}, \sigma_{n+1}\right]=0$ pour $1 \leq i \leq n$ et $d w\left(\sigma_{n+1}\right)=1$.

Considérons la matrice $\Lambda=\left(\Lambda_{i, j}\right)_{1 \leq i, j \leq n+1}$ définie comme l'evaluation des 1-formes $\theta_{i}=d L_{F}^{i-1} h$ pour $i=1:$ n et $\theta_{n+1}=d w$ sur le repère $\sigma=\left\{\sigma_{1}, \ldots, \sigma_{n}, \sigma_{n+1}\right\}$, c'est-à-dire $\Lambda_{i, j}=\theta_{i}\left(\sigma_{j}\right)$ pour $1 \leq i, j \leq n+1$. Par construction la matrice s'écrit comme suit

$$
\Lambda=\left(\begin{array}{cccccc}
0 & \cdots & 0 & 0 & 1 & * \\
\vdots & 0 & 0 & l \alpha_{n} & * & * \\
0 & 0 & \frac{l \pi}{\alpha_{1} \alpha_{2}} & * & * & * \\
0 & \frac{l \pi}{\alpha_{1}} & * & * & * & * \\
l \pi & * & * & * & * & * \\
0 & 0 & 0 & 0 & 0 & 1
\end{array}\right)
$$

Il est facile de voir qu'elle est inversible. Par consequent, on peut définir les multi-1 formes différentielles suivantes:

$$
\omega=\Lambda^{-1} \sigma=\left(\begin{array}{c}
\omega_{1} \\
\omega_{2} \\
\cdots \\
\omega_{n+1}
\end{array}\right)
$$

il est clair que : $\omega_{i} \sigma_{j}=\delta_{i}^{j}$, avec $\delta_{i}^{j}$ est le symbole de Kronecker. Soit $X$ et $Y$ deux champs de vecteurs $\left\{\sigma_{1}, \ldots, \sigma_{n}, \sigma_{n+1}\right\}$. On a :

$$
d \omega_{i}(X, Y)=L_{Y} \omega_{i}(X)-L_{X} \omega_{i}(Y)-\omega_{i}[X, Y]
$$

comme $\omega_{i}(X)$ et $\omega_{i}(Y)$ sont constantes alors

$$
d \omega_{i}(X, Y)=-\omega_{i}[X, Y]
$$


par conséquent $[X, Y]=0$ pour tous $X, Y$ si et seulement si $d \omega_{i}=0$ pour tous $1 \leq i \leq n+1$. D'où, grâce au lemme de Poincaré, il existe $\phi=\left(\phi_{1}, \ldots, \phi_{n+1}\right)$ tel que :

$$
\omega=D \phi:=\phi_{*}
$$

avec $D \phi$ la différentielle de $\phi$.

Nous considérons $\phi_{*}\left(\sigma_{i}\right)=\frac{\partial}{\partial z_{i}}$ pour $i=1$ : n et $\phi_{*}\left(\sigma_{i}\right)=\frac{\partial}{\partial \xi_{i}}$.

A présent, nous verrons comment $\phi_{*}$ transforme le champ de vecteurs $F$. Pour $i=1: n-1$, on $a$ :

$$
\begin{aligned}
{\left[\frac{\partial}{\partial z_{i}}, \phi_{*}(F)\right] } & =\left[\phi_{*}\left(\sigma_{i}\right), \phi_{*}(F)\right]=\phi_{*}\left[\sigma_{i}, F\right] \\
& =\alpha_{i} \phi_{*} \sigma_{i} \\
& =\alpha_{i} \frac{\partial}{\partial z_{i+1}}
\end{aligned}
$$

Alors par intégration, on a $\dot{z}_{i}=\alpha_{i}(y) z_{i}+\beta_{i}(y, w)$.

Nous terminons cette section par quelques remarques.

\section{Remarque 4. -}

- le calcul des fonctions $\alpha_{i}$ est détaillé dans (Zheng et al., 2007);

- pour le calcul des fonctions $l(w)$ et $\eta(w, y)$, les algorithmes de (Boutat, 2007; Boutat, Busawon, 2011) peuvent être utilisés

\section{Application au modèle SEIR}

Dans cette section, on utilisera l'algorithme présenté ci-dessus pour le calcul du changement de cordonnées transformant le modèle SEIR (6)-(8) sous la forme normale (12)-(16).

Les 1-formes (20) d'observabilité du système (6)-(8) sont données par:

$$
\begin{aligned}
& \theta_{1}=d x_{3} \\
& \theta_{2}=\varepsilon d x_{2}+\left(\gamma_{3}+2 \delta x_{3}\right) d x_{3} \\
& \theta_{3}=\varepsilon \beta x_{3} d x_{1}+\varepsilon\left(\gamma_{2}+\gamma_{3}+3 \delta x_{3}\right) d x_{2}+Q_{1} d x_{3}
\end{aligned}
$$

avec : $Q_{1}=\varepsilon \beta x_{1}+3 \varepsilon \delta x_{2}+\varepsilon q b+\gamma_{3}^{2}+6 \delta \gamma_{3} x_{3}+6 \delta^{2} x_{3}^{2}$

Un calcul direct donne le repère de Krener \& Isidori (21):

$$
\begin{aligned}
& \tau_{1}=\frac{1}{\varepsilon \beta x_{3}} \frac{\partial}{\partial x_{1}} \\
& \tau_{2}=u \tau_{1}+\frac{1}{\varepsilon} \frac{\partial}{\partial x_{2}} \quad \text { où } u=-b+\gamma_{3}+\left(\delta+\gamma_{1}\right) x_{3}+\varepsilon \frac{x_{2}}{x_{3}} \\
& \tau_{3}=-\frac{p b}{\varepsilon} \frac{\partial}{\partial x_{1}}-\left(L_{f} u\right) \tau_{1}+u \tau_{2}+\frac{1}{\varepsilon}\left(\gamma_{2}+\delta x_{3}\right) \frac{\partial}{\partial x_{2}}+\frac{\partial}{\partial x_{3}}
\end{aligned}
$$


puis, on vérifie si la condition de commutativité (22) est satisfaite:

$$
\begin{aligned}
& {\left[\tau_{1}, \tau_{2}\right]=\left[\tau_{1}, \tau_{3}\right]=0} \\
& {\left[\tau_{2}, \tau_{3}\right]=Q_{2} \tau_{1}+\frac{1}{x_{3}} \tau_{2}}
\end{aligned}
$$

$\operatorname{avec} Q_{2}=-\left(3 \delta+2 \gamma_{1}+\frac{2 \gamma_{2}-\gamma_{3}}{x_{3}}-3 \varepsilon \frac{x_{2}}{x_{3}^{2}}\right)$

Comme $\left[\tau_{2}, \tau_{3}\right] \neq 0$, c-a-d la condition de commutativité n'est pas satisfaite, alors le système (6)-(8) ne peut pas être transformé, mais on utilisera les $\tau_{i}$ pour construire le nouveau repère $\bar{\tau}$ donné dans (26). En se référant à (Zheng et al., 2007), on peut utiliser l'equation:

$$
\left[\tau_{2}, \tau_{3}\right]=\lambda(y) \tau_{2} \bmod \tau_{1}
$$

avec $\lambda(y)=\frac{d \alpha_{2}(y)}{d y} \frac{1}{\alpha_{2}(y)}$ pour determiner $\alpha_{2}(y)$. Alors, on a $\frac{d \alpha_{2}(y)}{d y} \frac{1}{\alpha_{2}(y)}=\frac{1}{x_{3}}=\frac{1}{y}$ ce qui donne $\alpha_{2}(y)=x_{3}$. On peut toujours supposer que $\alpha_{3}(y)=1$.

Par conséquent, on a $\alpha_{2}=x_{3}, \alpha_{1}=1$ et $\pi=\alpha_{2} \alpha_{2}=x_{3}$. Alors, à partir de (26) on obtient:

$$
\begin{aligned}
\bar{\tau}_{1} & =x_{3} \tau_{1}=\frac{1}{\varepsilon \beta} \frac{\partial}{\partial x_{1}} \\
\bar{\tau}_{2} & =\frac{1}{x_{3}}\left[\bar{\tau}_{1}, f\right]=\frac{1}{\varepsilon \beta x_{3}}\left(-b+\gamma_{1} x_{3}\right) \frac{\partial}{\partial x_{1}}+\frac{1}{\varepsilon} \frac{\partial}{\partial x_{2}} \\
\bar{\tau}_{3} & =\left[\bar{\tau}_{2}, f\right]=\left(-p b \beta+\frac{\left(-b+\gamma_{1} x_{3}\right)^{2}}{x_{3}}-b \varepsilon \frac{x_{2}}{x_{3}^{2}}\right) \bar{\tau}_{1} \\
& +\frac{1}{\varepsilon}\left(\gamma_{2}-b+\left(\delta+\gamma_{1}\right) x_{3}\right) \frac{\partial}{\partial x_{2}}+\frac{\partial}{\partial x_{3}}
\end{aligned}
$$

Un simple calcul nous donne

$$
\left[\bar{\tau}_{1}, \bar{\tau}_{2}\right]=\left[\bar{\tau}_{1}, \bar{\tau}_{3}\right]=0 \text { et }\left[\bar{\tau}_{2}, \bar{\tau}_{3}\right]=-2 \frac{b}{x_{3}^{2}} \bar{\tau}_{1}
$$

Comme $\left[\bar{\tau}_{2}, \bar{\tau}_{3}\right] \neq 0$, alors la condition (27) de commutativité n'est pas satisfaite. Cependant, en se référant au Lemme 3.1 dans (Boutat, Busawon, 2011), les conditions pour l'application de l'algorithme de la dynamique étendue sont satisfaites. Alors, nous chercherons une dynamique auxiliaire $\dot{w}=\eta(y, w)$ et une fonction $l(w)$ satisfaisant les conditions du théorème (2) énoncé ci-dessus.

Remarque 5. - D'après le Lemme 3.1 de (Boutat, Busawon, 2011) $\left[\bar{\tau}_{i}, \bar{\tau}_{n}\right]$ $\varrho(y) \bar{\tau}_{i-1} \in \operatorname{span}\left\{\bar{\tau}_{1}, \cdots, \bar{\tau}_{i-1}\right\}$ est une condition assurant l'existence de $\eta(y, w)$ et $l(w)$, avec $\varrho(y)$ est une fonction de $y$.

Par consequent, posons $\sigma_{1}=l(w) \bar{\tau}_{1}$, ce qui donne :

$$
\sigma_{1}=\frac{l}{\varepsilon \beta} \frac{\partial}{\partial x_{1}}
$$


Par induction, on a :

$$
\sigma_{2}=\frac{1}{x_{3}}\left[\sigma_{1}, F\right]=\frac{1}{x_{3}}\left(l H-\eta l^{\prime}\right) \sigma_{1}+\frac{l}{\varepsilon} \frac{\partial}{\partial x_{2}}
$$

où $H=\left(-b+\gamma_{1} x_{3}\right)$. Ensuite, on a:

$$
\begin{aligned}
\sigma_{3}= & -\frac{p b l}{\varepsilon} \frac{\partial}{\partial x_{1}}+\frac{\left(l H-\eta l^{\prime}\right)}{\varepsilon} \sigma_{2}-\frac{G}{\varepsilon l} \sigma_{1} \\
& +\frac{l\left(\gamma_{2}+q x_{3}\right)-\eta l^{\prime}}{\varepsilon} \frac{\partial}{\partial x_{2}}+l \frac{\partial}{\partial x_{3}}
\end{aligned}
$$

avec $G=\varepsilon^{2} b l \frac{x_{2}}{x_{3}}-\varepsilon^{2} l^{\prime} x_{2}\left(\frac{\eta^{\prime} x_{3}-\eta}{x_{3}^{2}}\right)+\eta\left(\frac{\varepsilon}{x_{3}}\left(l^{\prime} H-\eta l^{\prime \prime}\right)\right) \sigma_{1}$ et : $\eta^{\prime}=\frac{\partial \eta}{\partial x_{3}}$. Finalement, on obtient :

$$
\left[\sigma_{2}, \sigma_{3}\right]=2\left(\varepsilon^{2} l^{\prime} l \frac{\eta^{\prime} x_{3}-\eta}{x_{3}}-\frac{b \varepsilon^{2} l^{2}}{x_{3}^{2}}\right) \sigma_{1}
$$

On impose que

$$
\left[\sigma_{2}, \sigma_{3}\right]=0 \Longleftrightarrow l^{\prime} l\left(\eta^{\prime} x_{3}-\eta\right)-b l^{2}=0
$$

La solution de l'équation précédente est donnée par :

$$
l=e^{w} \text { et } \eta=\gamma_{1} x_{3}-b
$$

Alors le système dynamique étendu devient :

$$
F=\left\{\begin{array}{l}
\dot{x}_{1}=b-b x_{1}+\gamma_{1} x_{1} x_{3}-p b x_{2}-q b x_{3} \\
\dot{x}_{2}=\beta x_{1} x_{3}+\gamma_{2} x_{2}+\delta x_{2} x_{3}+q b x_{3} \\
\dot{x}_{3}=\varepsilon x_{2}+\gamma_{3} x_{3}+\delta x_{3}^{2} \\
\dot{w}=\gamma_{1} x_{3}-b \\
y=x_{3}
\end{array}\right\}
$$

Par l'application de l'algorithme précédemment décrit, et en remplaçant $l$ et $\eta$ par leur valeur touvée dans (34) on obtient :

$$
\begin{aligned}
\sigma_{1}= & \frac{e^{w}}{\varepsilon \beta} \frac{\partial}{\partial x_{1}}, \quad \sigma_{2}=\frac{e^{w}}{\varepsilon} \frac{\partial}{\partial x_{2}} \\
\sigma_{3}= & -\frac{e^{w}}{\varepsilon} p b \frac{\partial}{\partial x_{1}}+\frac{e^{w}}{\varepsilon}\left(\gamma_{2}+b+\left(\delta-\gamma_{1}\right) x_{3}\right) \frac{\partial}{\partial x_{2}} \\
& +e^{w} \frac{\partial}{\partial x_{3}}
\end{aligned}
$$


$\bar{C}$ e nouveau repère $\sigma$ vérifie la condition de commutativité, tels que :

$$
\left[\sigma_{1}, \sigma_{2}\right]=\left[\sigma_{1}, \sigma_{3}\right]=\left[\sigma_{2}, \sigma_{3}\right]=0
$$

Étant donné que le nouveau système, augmenté, est de dimension 4 , alors il faut trouver un quatrième champ de vecteurs $\sigma_{4}$ qui commute avec $\sigma_{1}, \sigma_{2}$ et $\sigma_{3}$ :

$$
\sigma_{4}=\frac{\partial}{\partial w}+x_{1} \frac{\partial}{\partial x_{1}}+\left(x_{2}+\frac{\delta-\gamma_{1}}{2 \varepsilon} x_{3}^{2}\right) \frac{\partial}{\partial x_{2}}+x_{3} \frac{\partial}{\partial x_{3}}
$$

on a $\left[\sigma_{4}, \sigma_{i}\right]=0$ pour $1 \leq i \leq 3$. A partir de là, il devient simple de calculer la matrice d'observabilité $\theta_{e}$, telle que :

$$
\theta_{e}=\left[d h, d L_{F} h, d L_{F}^{2}, d w\right]^{T}
$$

puis on obtient :

$$
\Lambda=\theta_{e} \sigma=\left(\begin{array}{cccc}
0 & 0 & e^{w} & x_{3} \\
0 & e^{w} & \Lambda_{23} & \Lambda_{24} \\
x_{3} e^{w} & e^{w}\left(\gamma_{2}+\delta x_{3}\right) & \Lambda_{33} & \Lambda_{34} \\
0 & 0 & 0 & 1
\end{array}\right)
$$

avec:

$$
\begin{aligned}
\Lambda_{23} & =e^{w}\left(b+\gamma_{2}+x_{3}\left(\delta-\gamma_{1}\right)\right) \\
\Lambda_{24} & =\varepsilon\left(x_{2}+\frac{1}{2 \varepsilon} x_{3}^{2}\left(\delta-\gamma_{1}\right)\right) \\
\Lambda_{33} & =e^{w}\left(\gamma_{2}+\delta x_{3}\right)\left(b+\gamma_{2}+x_{3}\left(\delta-\gamma_{1}\right)\right) \\
& +\varepsilon e^{w}\left(\beta x_{1}+\delta x_{2}+b q\right)-b p \beta x_{3} e^{w} \\
\Lambda_{34} & =\varepsilon x_{3}\left(\beta x_{1}+\delta x_{2}+b q\right) \\
& +\varepsilon\left(\gamma_{2}+\delta x_{3}\right)\left(x_{2}+\frac{1}{2 \varepsilon} x_{3}^{2}\left(\delta-\gamma_{1}\right)\right)+\beta \varepsilon x_{1} x_{3}
\end{aligned}
$$

Par un simple calcul, le changement de coordonnées est donné par sa différentielle comme suit :

$$
\begin{aligned}
\omega & =\Lambda^{-1} \theta_{e}=d z \\
& =d\left(\begin{array}{c}
\varepsilon \beta x_{1} e^{-w}+b p \beta x_{3} e^{-w} \\
\varepsilon x_{2} e^{-w}-\left(b+\gamma_{2}\right) x_{3} e^{-w}-\frac{1}{2}\left(\delta-\gamma_{1}\right) x_{3}^{2} e^{-w} \\
x_{3} e^{-w} \\
w
\end{array}\right)
\end{aligned}
$$


Finalement, la forme normale du système (1)-(5) est donnée par :

$$
\begin{aligned}
\dot{z}_{1} & =B_{1}(w, y) \\
\dot{z}_{2} & =y z_{1}+B_{2}(w, y) \\
\dot{z}_{3} & =z_{2}+B_{3}(w, y) \\
\dot{z}_{4} & =\gamma_{1} y-b \\
\bar{y} & =z_{3}=y e^{-w}
\end{aligned}
$$

avec :

$$
\begin{aligned}
B_{1}(y, w)= & -\left(\varepsilon \beta q b+b p \beta \gamma_{3}+b^{2} p \beta\right) e^{-w} x_{3}+ \\
& \left(b p \beta d-b p \beta \gamma_{1}\right) e^{-w} x_{3}^{2}+\varepsilon \beta b e^{-w} \\
B_{2}(y, w)= & \left(\varepsilon q b-\left(b+\gamma_{2}\right) \gamma_{3}-\left(b+\gamma_{2}\right) b\right) e^{-w} x_{3}+ \\
& \left(-\beta p b-\left(b+\gamma_{2}\right) d+\left(b+\gamma_{2}\right) \gamma_{1}\right. \\
& \left.-\left(\delta-\gamma_{1}\right) \gamma_{3}-\frac{\delta-\gamma_{1}}{2} b\right) e^{-w} x_{3}^{2}+ \\
& \left(-\left(\delta-\gamma_{1}\right) d+\frac{\delta-\gamma_{1}}{2} \gamma_{1}\right) e^{-w} x_{3}^{3} \\
B_{3}(y, w)= & \left(2 b+\gamma_{2}+\gamma_{3}\right) e^{-w} x_{3}+\frac{1}{2}\left(d+\delta-2 \gamma_{1}\right) e^{-w} x_{3}^{2}
\end{aligned}
$$

\section{Résultats de simulation}

Les valeurs des paramètres utilisés dans le modèle épidémique sont : $N=141, b=$ $0.221176 / N, d=0.002, \quad p=0.8, q=0.95, \quad \beta=0.05, \quad \varepsilon=0.05, \quad r=0.003$. $\mathrm{S}(0)=140 ; \mathrm{E}(0)=0.01 ; \mathrm{I}(0)=0.02 ; \mathrm{N}(0)=141$.

Pour rendre la simulation proche de la réalité, on a ajouté un bruit blanc à la sortie du modèle $I(t)$ et on a pris des conditions initiales différentes entre le modèle (1)-(5) et l'observateur. En se référant aux figures (Fig.2, Fig.4, Fig.6) la convergence des estimations est rapide mais des oscillations apparaissent due au choix du gain. Pour la simulation, on a choisi $\rho=0.35$, ce qui satisfait le compromis entre la stabilité et la convergence de l'estimateur.

\section{Conclusion}

Ce papier présente une nouvelle forme d'observabilité étendue et dépendante de la sortie. Des condition suffisantes à l'existence du changement de coordonnées sont établies pour une classe de systèmes non linéaires. Cette nouvelle forme permet l'utilisation de l'observateur à grand gain. 


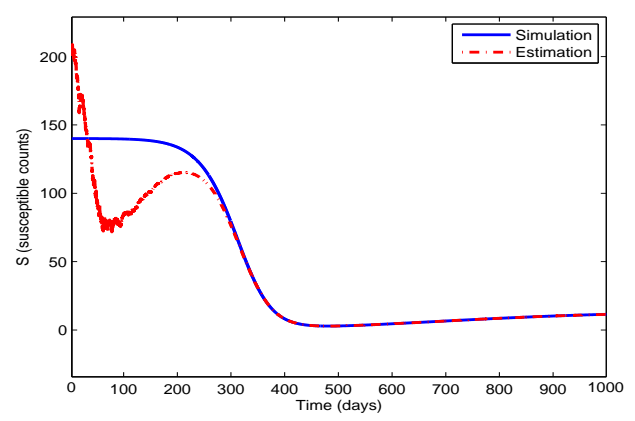

Figure 2. Estimation de la population susceptible.

Figure 1.

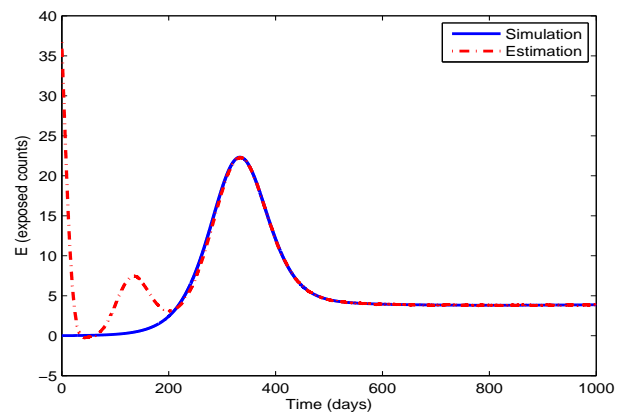

Figure 4. Estimation de la population exposée.

Figure 3.

(Chapter head:)*

\section{Références}

Back J., Yu K. T., Seo J. H. (2006). Dynamic observer error linearization. Automatica, vol. 42, $n^{\circ} 12$, p. 2195-2200.

Bestle D., Zeitz M. (1983). Canonical form observer design for nonlinear time varying systems. International Journal of Control, vol. 38, p. 419-431.

Boutat D. (2007). Geometrical conditions for observer error linearization via $\int 0,1, \ldots,(N-2)$. In 7th ifac symposium on nonlinear control systems nolcos'07,.

Boutat D., Benali A., Hammouri H., Busawon K. (2009). New algorithm for observer error linearization with a diffeomorphism on the outputs. Automatica, vol. 45, no 10, p. $2187-$ 


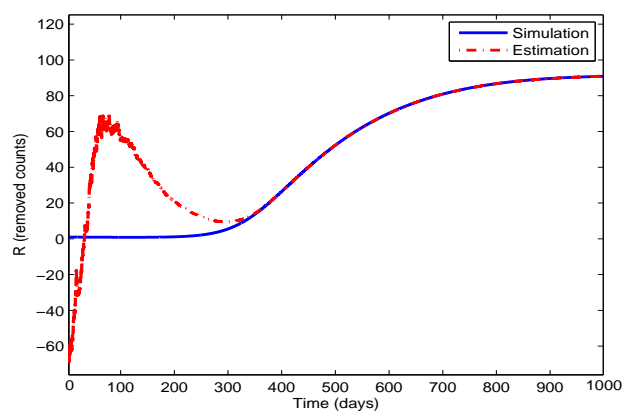

Figure 6. Estimation de la population traitée.

Figure 5.

2193.

Boutat D., Busawon K. (2011). On the transformation of nonlinear dynamical systems into the extended nonlinear observable canonical form. International Journal of Control, vol. 84, $\mathrm{n}^{\circ} 1$, p. 94-106.

Boutat D., Zheng G., Barbot J., Hammouri H. (2006). Observer error linearization multi-output depending. In the 45th ieee conference on decision and control, p. 5394-5399.

Busawon K., Farza M., Hammouri H. (1998). Asimple observer for a class of nonlinear systems. Applied Mathematics Letters, vol. 11, n 3, p. 27-31.

Hou M., Pugh A. (1999). Observer with linear error dynamics for nonlinear multi-output systems. Systems \& Control Letters, vol. 37, p. 1-9.

Iggidr A., Kamgang J., Sallet G., Tewa J. (2006). Global analysis of new malaria intrahost models with a competitive exclusion principle. SIAM Journal on Applied Mathematics, p. 260-278.

Jouan P. (2003). Immersion of nonlinear systems into linear systems modulo output injection. SIAM Journal on Control and Optimisation, vol. 41(6), p. 1756-1778.

Kazantzis N., Kravaris C. (1998). Nonlinear observer design using lyapunoves auxiliary theorem. Systems \& Control Letters, vol. 34, no 5, p. 241-247.

Keller H. (1987). Nonlinear observer design by transformation into a generalized observer canonical form. International Journal of Control, vol. 46(6), p. 1915-1930.

Krener A., Isidori A. (1983). Linearization by output injection and nonlinear observers. Systems \& Control Letters, vol. 3, n 1, p. 47-52.

Krener A., Respondek W. (1985). Nonlinear observers with linearizable error dynamics. SIAM Journal on Control and Optimization, vol. 23, n 2, p. 197-216.

Lopez M., Plestan F., Glumineau A. (1999). Linearization by completely generalized input ouput injection. Kybernetika, vol. 35, p. 793-802. 
Lynch A., Bortoff S. (2001). Nonlinear observers with approximately linear error dynamics: the multivariable case. IEEE Transactions on Automatic Control, vol. 46, nº 6, p. 927-932.

Marino R., Tomei P. (1996). Nonlinear control design: geometric, adaptive and robust (P. H. I. U. Ltd, Ed.).

Noh D., Jo N., Seo J. (2004). Nonlinear observer design by dynamic observer error linearization. IEEE Transactions on Automatic Control, vol. 49, nº 10, p. 1746-1753.

Phelps A. (1991). On constructing nonlinear observers. SIAM Journal on Control and Optimmisation, vol. 29, p. 516-534.

Respondek W., Pogromsky A., Nijmeijer H. (2004). Time scaling for observer design with linearizable error dynamics. Automatica, vol. 40 (2), p. 277-285.

Rudolph J., Zeitz M. (1994). A block triangular nonlinear observer normal form. Systems \& Control Letters, vol. 23, $\mathrm{n}^{\circ}$ 1, p. 1-8.

Wang Y., Lynch A. (2009). Observer design using a generalized time-scaled block triangular observer form. Systems \& Control Letters, vol. 58, p. 346-352.

Xia X., Gao W. (1989). Nonlinear observer with linearizable error dynamics. SIAM Journalon Control and Optimization, vol. 27, p. 199-216.

Yang J., Back J., Seo J., Shim H. (2010). Reduced-order dynamic observer error linearization. In Nonlinear control systems, p. 915-920.

Zheng G., Boutat D., Barbot J. (2007). A single output dependent observability normal form. SIAM Journal on Control and Optimization, vol. 46, n 6, p. 2242-2255. 